\title{
SIMILARITY OF A LINEAR STRICT SET-CONTRACTION AND THE RADIUS OF THE ESSENTIAL SPECTRUM
}

\author{
MAU-HSIANG SHIH
}

\begin{abstract}
If $A$ is a bounded linear operator on a Hilbert space, define $r_{e}(A)$, the essential spectral radius of $A$, by $r_{e}(A):=\sup \{|\lambda|: \lambda \in \operatorname{ess}(A)=$ essential spectrum of $A\}$.

It is shown that

$r_{e}(A)=\inf \left\{\alpha\left(S^{-1} A S\right) \mid S: H \rightarrow H\right.$ is a bounded invertible linear map $\}$,

where $\alpha$ is the Kuratowski measure of noncompactness. As a consequence, a charcterization of the similarity of a linear strict set-contraction is obtained.
\end{abstract}

1. This paper applies a classical result of Rota with known results about Browder's essential spectrum to give a new formula for the radius of the essential spectrum of a bounded linear operator in Hilbert space.

Throughout the paper, $H$ will denote a complex Hilbert space with norm $\|\cdot\|$. By an operator we always mean a bounded linear transformation on $H$. The identity operator is denoted by $I$. The spectrum of an operator $A$ is denoted by $\sigma(A)$ and the spectral radius is denoted by $r(A)$. Recall that an operator $A$ is called a strict contraction if $\|A\|<1[2$, p. 82]. The classical result of Rota's similarity theorem $[6 ; 2$, p. 81$]$ asserts that an operator on $H$ is similar to a strict contraction if and only if its spectrum is included in the interior of the unit disc. There is an elegant quantitative version of Rota's theorem [2, p. 77]; it asserts that the spectral radius of $A$ is always equal to the infimum of norms of all conjugates (i.e., transformation by similarities) of $A$. Seeking to characterize the similarity of a linear strict set-contraction (the perturbation of a strict contraction by a compact operator is a strict set-contraction), we may therefore raise the question: What kind of spectrum included in the interior of unit disc has to be similar to a linear strict set-contraction? Thanks to the works of Kuratowski [3], Browder [1], Nussbaum [5], and Leggett [4], we are capable of solving this full problem. Our main results are proved in $\S 3$ and $\S 2$ contains some preliminary notions and lemmas.

The author wishes to thank the referee for his valuable suggestions.

2. We first recall the "measure of noncompactness," a notion which was introduced by Kuratowski in 1930 [3].

Let $\Omega$ be a nonempty subset of $H$. Kuratowski [3] defined the measure of noncompactness of $\Omega$, in symbols $\alpha(\Omega)$, to be

$$
\begin{aligned}
\inf \{\varepsilon>0: & \Omega \text { can be covered by a finite number } \\
& \text { of sets of diameter less than or equal to } \varepsilon\} .
\end{aligned}
$$

Received by the editors March 24, 1986.

1980 Mathematics Subject Classification (1985 Revision). Primary 47A15, 47B37.

Key words and phrases. Essential spectrum, $k$-set-contraction, measure of noncompactness, similarity of operators. 
It follows immediately that a subset $\Omega$ of $H$ has a compact closure if and only if $\alpha(\Omega)=0$. Closely associated with the notion of the measure of noncompactness is the concept of " $k$-set-contraction," defined as follows. Let $T$ be a continuous map from $\Omega$ into $H$; then $T$ is called a $k$-set-contraction if there exists a constant $k \geq 0$ such that for any nonempty bounded set $D \subset H$ we have $\alpha[T(D)] \leq k \alpha(D)$. If $T$ is a $k$-set-contraction, Nussbaum [5] defined the measure of noncompactness of $T$, in symbols $\alpha(T)$, to be

$$
\inf \{k>0: T \text { is a } k \text {-set-contraction }\} .
$$

If $\alpha(T)<1$, then $T$ is called a strict set-contraction. If $A:=C+S$, where $C$ is a compact operator and $S$ a strict contraction, then $A$ is a strict set-contraction.

We may recall another notion introduced by Browder [1], that of the essential spectrum of an operator. Browder defined the essential spectrum of an operator $A$, in symbols ess $(A)$, to be the set of $\lambda \in \sigma(A)$ such that at least one of the following conditions holds:

(i) $R(\lambda I-A)$, the range of $\lambda I-A$, is not closed.

(ii) $\lambda$ is a limit point of $\sigma(A)$.

(iii) $\bigcup_{\nu=1}^{\infty} N(\lambda I-A)^{\nu}$ is infinite dimensional, where $N(\lambda I-A)^{\nu}$ denotes the null space of $(\lambda I-A)^{\nu}$.

If $A$ is a an operator on $H$, define $r_{e}(A)$, the essential spectral radius of $A$, by

$$
r_{e}(A):=\sup \{|\lambda|: \lambda \in \operatorname{ess}(A)\} .
$$

Nussbaum proved in [5] that

$$
r_{e}(A)=\lim _{n \rightarrow \infty}\left(\alpha\left(A^{n}\right)\right)^{1 / n}
$$

Note that the above formula should be extended to read

$$
r_{e}(A)=\inf _{n \geq 1}\left(\alpha\left(A^{n}\right)\right)^{1 / n} \leq \alpha(A) .
$$

For an operator $A$, we observe that $A$ is a $\|A\|$-set-contraction and hence $\alpha(A) \leq$ $\|A\|$.

The following lemmas will be needed in the proofs of our results. When we say an operataor is finite dimensional, we shall mean its range is finite dimensional.

Lemma 1 (Nussbaum [5]). Let $A$ be an operator on $H$ and $r>r_{e}(A)$. Then there exists a finite dimensional operator $F$ on $H$ such that $\sigma(A+F) \subset$ $\{\lambda \in \mathbf{C}:|\lambda| \leq r\}$.

LEMMA 2. Similar operators on $H$ have the same essential spectrum.

ProOF. Let $A$ be an operator on $H$ and $B=P^{-1} A P$ for some invertible operator $P$ on $H$. Then the conclusion follows from the following identities. (The identity (ii) below is a known result.)

(i) $P^{-1}[R(\lambda I-A)]=R(\lambda I-B), P^{-1}[\operatorname{cl}(\lambda I-A)]=\operatorname{cl}(\lambda I-B)$, where $\operatorname{cl}(\lambda I-A)$ denotes the closure of $(\lambda I-A)(H)$.

(ii) $\sigma(A)=\sigma(B)$.

(iii) $P^{-1}\left[N(\lambda I-A)^{\nu}\right]=N(\lambda I-B)^{\nu}$ for each positive integer $\nu$. 
3. Our main result is the following:

THEOREM 1. Let $A$ be an operator on $H$. Then

$$
r_{e}(A)=\inf \left\{\alpha\left(S^{-1} A S\right) \mid S: H \rightarrow H \text { is a bounded invertible linear map }\right\} .
$$

Proof. Lemma 2 implies that if $S: H \rightarrow H$ is bounded and invertible,

$$
r_{e}(A)=r_{e}\left(S^{-1} A S\right) \leq \alpha\left(S^{-1} A S\right)
$$

Thus one has

$$
r_{e}(A) \leq \inf \left\{\alpha\left(S^{-1} A S\right) \mid S \text { is one-one, onto, and linear }\right\} .
$$

To prove the opposite inequality, take $\varepsilon>0$ and use Lemma 1 to find a finitedimensional operator $F$ such that

$$
r(A+F) \leq r_{e}(A)+\varepsilon / 2 .
$$

By Rota's theorem, there exists $S$ such that

$$
\left\|S^{-1}(A+F) S\right\| \leq r(A+F)+\varepsilon / 2 \leq r_{e}(A)+\varepsilon .
$$

Finally we have (using basic properties of the seminorm $\alpha$ )

$$
\left\|S^{-1}(A+F) S\right\| \geq \alpha\left(S^{-1}(A+F) S\right)=\alpha\left(S^{-1} A S+S^{-1} F S\right)=\alpha\left(S^{-1} A S\right) .
$$

Here we have used that $\|B\| \geq \alpha(B)$ and $\alpha(B+C)=\alpha(B)$ for any compact linear map $C$. This proves that

$$
\alpha\left(S^{-1} A S\right) \leq r_{e}(A)+\varepsilon
$$

and since $\varepsilon>0$ was arbitrary, the proof is complete.

THEOREM 2. Let $A$ be an operator on $H$. Then $A$ is similar to a linear strict set-contraction if and only if $r_{e}(A)<1$.

ProOF. This is immediate from Lemma 2 and Theorem 1 above.

\section{REFERENCES}

1. F. E. Browder, On the spectral theory of elliptic differential operators. I, Math. Ann. 142 (1961), 22-130.

2. P. R. Halmos, A Hilbert space problem book, 2nd ed., revised and enlarged, Springer-Verlag, New York, Heidelberg, and Berlin, 1982.

3. C. Kuratowski, Sur les espaces complets, Fund. Math. 15 (1930), 301-309.

4. R. Leggett, Remarks on set-contractions and condensing maps, Math. Z. 132 (1973), 361-366.

5. R. D. Nussbaum, The radius of the essential spectrum, Duke Math. J. 38 (1970), 473-478.

6. G.-C. Rota, On models for linear operators, Comm. Pure Appl. Math. 8 (1960), 469-472.

Department of Mathematics, Chung Yuan University, Chung-Li, Taiwan, REPUBLIC OF CHINA 